\title{
EXPERIENCIA SUBJETIVA DE ESTUDIANTES Y DOCENTES QUE VIVENCIAN UN CAMBIO CURRICULAR ${ }^{1}$
}

\author{
SUBJECTIVE EXPERIENCE OF STUDENTS AND TEACHERS \\ WHO EXPERIENCE A CURRICULUM CHANGE
}

\author{
EDITH Rivas R.* \\ Luis Bustos M.** \\ ANGÉlica Rivas L. ${ }^{* * *}$ \\ Ximena Lagos P. ${ }^{* * * *}$ \\ Silvia SAN MARTín G. ${ }^{* * * *}$
}

\begin{abstract}
RESUMEN
Objetivo: Conocer la experiencia subjetiva de estudiantes y docentes respecto del cambio curricular. Metodología: Estudio transversal, muestra de 169 estudiantes de cuarto año curricular y 32 docentes. Información recogida en cuestionario "Inquietudes y procedimientos para adoptar Innovaciones Educativas" (IPIE) validado y adaptado al estudio. Análisis descriptivo. Estudio respetó normas éticas y consentimiento informado. Resultados: 26,0\% de alumnos correspondió a Tecnología Médica; 22,5\% Enfermería y Nutrición, respectivamente, y 29,0\% Obstetricia. Edad media de 21,3 $\pm 1,43$ años. Mujeres 68,1\%. En “Información”, los estudiantes mostraron el promedio más alto en el ítem "Quisiera saber lo que otros están haciendo en esta área" $(4,1 \pm 0,9)$, y los docentes en "Me gustaría saber si se contará con los recursos para mantener esta innovación en un futuro inmediato" $(4,7 \pm 0,4)$. En "Funcionalidad", promedio más alto correspondió a "Me gustaría saber qué es lo que esta innovación va a requerir para mantenerla en el futuro" $(4,3 \pm 0,8)$ y el más bajo en "Estoy totalmente ocupado/a en otras cosas, para mí la innovación no es prioridad" $(2,4 \pm 1,4)$. En "Resultados", los docentes presentaron el promedio más alto en "Me interesa evaluar mi impacto sobre los estudiantes" y "Quisiera que otros equipos de docentes se motiven con los logros que tenemos en este proceso de innovación curricular" $(4,6 \pm 0,4 ; 4,6 \pm 0,4)$. Conclusiones: Estudiantes y docentes se mostraron conformes con la experiencia del cambio curricular. Aún existen dudas respecto de cómo este cambio afectará las competencias del estudiantado y la sustentabilidad en el tiempo. Es necesario continuar esta línea investigativa durante el ejercicio profesional de egresados.
\end{abstract}

Palabras clave: Currículum, educación en enfermería, educación basada en competencias.

\footnotetext{
ABSTRACT

Objective: To determine the subjective experience of students and teachers on curriculum change.Methodology: Cross-sectional study in a sample of 169 Regular fourth-year students and 32 teachers. Information collected in the survey "Concerns and procedures to adopt educational innovations" (IPIE) validated and adapted to the study. Descriptive analysis with means, medians, ranges, percentages and averages compared with t-test and ANOVA. Study respected ethical and informed consent. Results: $26.0 \%$ of students accounted for Medical Technology, Nursing and Nutrition 22.5\%, 29.0\% respectively and Obstetrics. Mean age $21.3 \pm 1.43$ years.

${ }^{1}$ Proyecto Financiado por la Dirección de Investigación de la Universidad de La Frontera No DI08-0070.

*Enfermera, Universidad de La Frontera, Temuco. Departamento de Pediatría Cirugía Infantil, Email: erivas@ufro.cl

** Bioestadístico. Universidad de La Frontera, Temuco. Email: lbustos@ufro.cl

${ }^{* * *}$ Enfermera. Docente Universidad Autónoma de Chile. Temuco. Email: ayrivasl@gmail.com

${ }^{* * * *}$ Enfermera. Docente Universidad de La Frontera. Email: xlagos@ufro.cl

${ }^{* * * * *}$ Enfermera. Docente Universidad de La Frontera. Email: ssanmart@ufro.cl
} 
$68.1 \%$ women. In "Information", students showed the highest average item "I want to know what others are doing in this area" (4.1 \pm 0.9$)$, and teachers in "I wonder if they have the resources to keep this innovation in the immediate future " $(4.7 \pm 0.4)$. In "Functionality" highest average corresponded to "I wonder what this innovation will be required to keep it in the future" ( $4.3 \pm 0.8)$ and lowest in the scale "I am fully occupied / to other things, for innovation is not my priority " $(2.4 \pm 1.4)$. In teaching, in "Results" highest average "I am interested in evaluating my impact on students" and "I want other teachers to motivate teams with the achievements we have in this curricular innovation process" (4.6 $\pm 0,4,4.6 \pm 0.4)$. Conclusions: students and teachers were satisfied with the experience of curriculum change. Still, there are doubts about how this will affect the student skills and sustainability over time. It is necessary to continue this line of research for the professional practice of graduates, to objectively assess the skills of the new student-centered curriculum.

Key words: Curriculo, nursing education, competency-based education.

Fecha recepción: 06/12/11 Fecha aceptación: 19/12/12

\section{INTRODUCCIÓN}

La calidad de la Educación Superior es un imperativo estratégico para el desarrollo de una nación, razón por la cual es una temática de preocupación constante a nivel ministerial, universitario, particular y privado, debido a que recae sobre las entidades formadoras la responsabilidad en la formación de recursos humanos del más alto nivel y la creación, desarrollo, transferencia y adaptación de tecnología para responder adecuadamente a los requerimientos de una sociedad cambiante (1-3).

Desde fines del siglo XX, la educación, a nivel mundial, ha experimentado un proceso de construcción y reconstrucción. Autores como Bendersky, entre otros, señalan que existe un largo camino por recorrer para que la educación nacional se pueda insertar en el mundo global. De esta forma, se genera una cultura de aprendizaje permanente, creando articulaciones necesarias dentro del sistema educacional y en el mundo laboral, social y cultural (4).

Chile posee un sistema de educación superior que ha alcanzado un relativo grado de madurez, no exento de mejora, con profundos desafíos que abordar en las próximas décadas. Se debe considerar que el solo acceso no garantiza equidad, es menester asegurar una permanencia adecuada y evitar la deserción. Asimismo, generar condiciones de aprendizaje de calidad y relevancia, independientemente de la modalidad de formación y del tipo de propiedad de estas instituciones (4), lo cual debe dar respuesta a una acreditación de calidad, que incluye ofrecer el máximo de condiciones posibles y factibles para una óptima formación profesional (5).

La Facultad de Medicina de la Universidad de La Frontera, como una forma de responder a las exigencias de formación de profesionales necesarios para este mundo globalizado, el año 2002 dio inicio a un proceso de innovación en la formación de sus estudiantes, proyecto que involucró a las carreras de Enfermería, Kinesiología, Medicina, Nutrición y Dietética, Obstetricia Puericultura y Tecnología Médica y que finalizó el año 2007, incluyendo el desarrollo e implementación de un cambio curricular, que respondiera a las estrategias metodológicas, que permitan el desarrollo de las competencias tanto genéricas como profesionales guiadas desde el aprendizaje centrado en el estudiante (6).

El estudio de esta innovación requirió considerar impactos, análisis interno del modo de cómo ha sido llevada a la práctica y análisis evaluativo de los factores asociados 
con la praxis del currículum, constituyéndose la innovación de la evaluación de los procesos de manera explícita e implícita en la piedra angular, manifestándose como sólidas razones para involucrarse en la innovación curricular, el avance de las políticas de salud, el mercado, la demanda de profesionales para la atención primaria y la formación de profesionales para la atención terciaria de salud y especialidad.

El proceso fue llevado a cabo adaptando la metodología de Propuesta Curricular por Competencias para el pregrado médico realizada por Bandaranayake (7), donde lo primero consistió en determinar lo que el egresado debería ser capaz de realizar, para posteriormente obtener información acerca de cada responsabilidad, dividir cada tarea comprometida, separar cada tarea en sus componentes, en las áreas del saber, del hacer y el demostrar, categorizando el contenido seleccionado, eligiendo los métodos más adecuados y planificando su evaluación.

Una de las estrategias pedagógicas centrales fue apostar por un cambio de paradigma hacia el aprendizaje basado en problemas, concebido como una metodología centrada en la investigación y análisis reflexivo para dar soluciones a situaciones puntuales, lo cual asegura que los protagonistas de estas transformaciones efectivamente cambien sus discursos. De esta forma, se propuso la necesidad de implementar cambios en todas las etapas del proceso de enseñanza/aprendizaje, incluyendo orientaciones en el área de evaluación (8). Se estableció una formación basada en prioridades de salud, temas prevalentes, que en conjunto al coherente cambio de paradigma educativo se diseñó nuevas herramientas para evaluar los resultados del aprendizaje (9). El propósito y fines de la evaluación denotaron especial interés y fueron mencionados como de importancia cardinal, así se refuerza la idea de la importancia de los cambios curriculares, que tienen relación con la evaluación de los aprendizajes y éstos, a su vez, con las necesidades del mundo ac- tual y la tecnología, como un medio de ayuda para la comunicación y el logro de calidad en la educación.

El proceso contó con un cuerpo de profesores perfeccionados, lo que reafirma la necesidad de contemplar sólidos agentes educadores, ya que sin ellos no se puede implementar ningún cambio curricular significativo (10), asegurando la relación entre el nuevo rol del docente y los desafíos en la docencia, característica innovadora, dado el tradicionalismo y carácter conservador de las universidades de América Latina (11). Al respecto, es menester que el conjunto de docentes asuma las ideas del proyecto institucional, sobre las materias y el grado de responsabilidad que les corresponde, ello simboliza extender su preocupación al interés por mejorar el desempeño en función de las consecuencias que tiene para los estudiantes, lo que significa alcanzar un mayor compromiso con los resultados que se logren en materia de aprendizaje. Esta situación implica establecer sistemas de evaluación que den cuenta de las necesidades reales del tipo de docencia y docente requerido por la unidad (perfil del docente) y que se registre inicialmente en el proceso docente el nivel del producto alcanzado y luego el impacto del proceso en su conjunto en la formación y desempeño de los profesionales en el ámbito del trabajo (11).

En relación a la evaluación de programas, se expone que en los Estados Unidos, a tres años de iniciar un currículo innovado, se preocuparon por la evaluación y validación, incluyendo técnicas de evaluación del desempeño diseñadas por los docentes (12).

Por lo anteriormente expuesto se hace imprescindible evaluar el desarrollo vivido por estudiantes y docentes, de manera de dar respuesta a la pregunta de investigación: ¿Cuál es la experiencia subjetiva de los estudiantes y académicos respecto de la innovación curricular en la Facultad de Medicina de la Universidad de La Frontera?, a fin de construir una visión de la situación actual, la 
cual no ha estado exenta de inquietudes, interrogantes, en proporción al cambio de las nuevas formas curriculares, siendo manifestado en los procesos de la evaluación de docentes y estudiantes (13). El presente trabajo tuvo como objetivo conocer la experiencia subjetiva de estudiantes y docentes, respecto del cambio curricular, en las carreras de Enfermería, Kinesiología, Nutrición y Dietética, Obstetricia y Puericultura y Tecnología Médica de la Facultad de Medicina de la Universidad de La Frontera, años 2009-2010.

\section{MATERIAL Y MÉTODO}

Estudio descriptivo, transversal. Se obtuvo una muestra no probabilística, por conveniencia, de 169 estudiantes que cursaban cuarto año curricular y 32 docentes de las carreras de Enfermería, Nutrición y Dietética, Obstetricia y Puericultura y Tecnología Médica, que aceptaron bajo consentimiento informado su inclusión en el estudio, respetando los principios éticos y criterios de rigor de Exequiel Emmanuel (14), proyecto que contó con aceptación del Comité de Ética.

En la recolección de la información, realizada en dependencias de la Universidad, se utilizó el cuestionario Inquietudes y procedimientos para adoptar Innovaciones Educativas (IPIE), adaptado idiomáticamente al estudio y validado por expertos, que incorporó las dimensiones: "Información", "Personal o de Proceso" para los estudiantes, e "Información”, "Personal-proceso", "Funcionalidad", "Disposición" y "Resultados" para los docentes, evaluados en escala tipo Likert, con intervalo de 1 a 5, de menor a mayor valoración, donde 1 es muy en desacuerdo y 5 muy de acuerdo.

El ingreso de datos se realizó en planilla Excel y el procesamiento con el Programa STATA 11.0v. Se realizó análisis descriptivo a través de tablas estadísticas, medias, medianas, mínimo, máximo y porcentajes.

\section{RESULTADOS}

En una muestra de 169 estudiantes de la Facultad de Medicina, el 26,0\% fueron de Tecnología Médica, 22,5\% Enfermería, 22,5\% Nutrición y 29,0\% Obstetricia; sus edades fluctuaron entre 19 y 28 años, media 21,3 $\pm 1,43$. Mujeres $68,1 \%$ y el $72,8 \%$ no había repetido asignatura.

En la muestra de 32 docentes, $81,3 \%$ eran mujeres. El 40,6\% de los docentes presentó edad entre 31 y 40 años y los tramos de edad menor a 30 años y entre 45 y 50 años, obtuvo una ponderación de 21,9\%. El 15,6\% tenía entre 51 y más años. El promedio de experiencia laboral fue de 12,4 años con DS 7,21, con un mínimo de 2 años y máximo de 34 años.

En las dimensiones "Información” y "Personal - proceso", se describen resultados en estudiantes y docentes. Y en las dimensiones "funcionalidad, disposición y resultados" se narran los resultados en docentes.

En la dimensión "Información” el promedio más alto se obtuvo en el ítem "Quisiera saber lo que otros están haciendo en esta área" $(4,1 \pm 0,9)$, y en los docentes "Me gustaría saber si se contará con los recursos para mantener esta innovación en un futuro inmediato" $(4,7 \pm 0,4)$. El promedio más bajo corresponde en estudiantes y docentes al ítem "No me interesa saber más de esta innovación" ( $1,8 \pm 1,0 ; 1,3 \pm 1,0)$. Generan inquietud los dominios "Ni siquiera sé en qué consiste esta innovación" y "Sé muy poco del proceso de innovación" expresados en los docentes $(1,9 \pm 1,4 ; 2,0 \pm 1,4)$ (Tabla 1$)$.

En los estudiantes, la dimensión "Personal - proceso" logró el promedio más alto en el ítem "Me inquieta que no pueda manejar todo lo que esta innovación requiere" $(4,4 \pm 0,6)$, y en los docentes "Me gustaría hablar de la posibilidad de implementar mejor esta innovación" $(4,0 \pm 0,8)$. El promedio más bajo se obtuvo en estudiantes y docentes en la dimensión "Conozco otras estrategias me- 
todológicas medios que podrían funcionar mejor" $(2,5 \pm 1,2 ; 2,8 \pm 1,2)$ (Tabla 1$)$.

En "Funcionalidad", el promedio más alto correspondió al ítem "Me gustaría saber qué es lo que esta innovación va a requerir para mantenerla en el futuro" $(4,3 \pm 0,8)$ y el promedio más bajo al ítem "Estoy totalmente ocupado/a en otras cosas, para mi la innovación no es prioridad" $(2,4 \pm 1,4)$ (Tabla 2$)$.

En "Disposición", el promedio más alto correspondió al ítem "Quisiera usar la opinión de los estudiantes para mejorar el proceso de innovación" $(4,4 \pm 0,7)$ y el promedio más bajo a "Quisiera saber cómo cambiar mi rol para colaborar en el proceso de innovación" $(3,7 \pm 1,2)$ (Tabla 2).

En "Resultados", el promedio más alto correspondió a los ítemes "Me interesa evaluar mi impacto sobre los estudiantes" y "Quisiera que otros equipos de docentes se motiven con los logros que tenemos en este proceso de innovación curricular" $(4,6 \pm 0,4 ; 4,6 \pm 0,4)$ y el promedio más bajo al ítem "Esta innovación no me inquieta" $(1,9 \pm 1,3)$ (Tabla 2$)$.

\section{DISCUSIÓN Y CONCLUSIÓN}

Existe consenso a nivel internacional que se debe dar respuesta a las necesidades de la globalización actual. En concordancia, la Facultad de Medicina (UFRO) intenta responder a las demandas de salud como al avance científico y nuevas tecnologías, iniciando un proceso de cambio curricular, basado en competencias enfocadas hacia los futuros problemas que abordarán los nuevos profesionales. De esta forma ha realizado un cambio de paradigma hacia el aprendizaje basado en problemas prioritarios de salud, abordando temas prevalentes y emergentes, que serán situaciones reales que el futuro profesional deberá enfrentar.

En este contexto, se puede obtener modelos de desarrollo de criterios para lograr un proceso enseñanza-aprendizaje de cali- dad, idea que se refleja en la preocupación expresada tanto por estudiantes como por los docentes, interesados en obtener mejores resultados con la innovación (10).

En este proceso de innovación existen cambios de roles, donde se gestan situaciones críticas, lo que impone mayor estrés sobre la institución. Sin embargo, los resultados obtenidos en este estudio revelan que existe gran preocupación de un grupo importante de académicos respecto de cómo cambiar su forma de enseñar, e invertir el tiempo que deben ocupar trabajando en las metodologías. Por otra parte, se ha visualizado en otros estudios resultados similares donde la inquietud de los académicos respecto a no poder manejar todo lo que la innovación requiere (15) constituye un eje transversal.

La innovación de un currículum tiene estrecha relación con la formación de sus docentes, proposición que concuerda con el trabajo efectuado en la Facultad de Medicina, que previo a la innovación planteada los profesionales se han perfeccionado en la Universidad de Mc Master, Canadá, grupo que por más de una década ha estado formando a los académicos de su Facultad para la innovación (5). De esta manera, se contribuye a que el currículo asegure a los estudiantes habilitaciones sobre las competencias, en sus distintos niveles, evidenciándose en el perfil de egreso (16).

Todo cambio "per se" requiere de una evaluación periódica, basada en el desempeño de los estudiantes ante actividades y problemas relacionados a su contexto profesional (17), además en el diseño curricular deben establecerse pautas evaluadoras de la gestión institucional, en relación al currículo y el desempeño del docente con base en tres tipos generales de valoración: autoevaluación, coevaluación y heteroevaluación. La evaluación utilizada en el currículo innovado está en completo acuerdo a lo planteado por Rial (17), donde en cada módulo quedan consignados los tipos de evaluación y criterios para llevarla a cabo. 


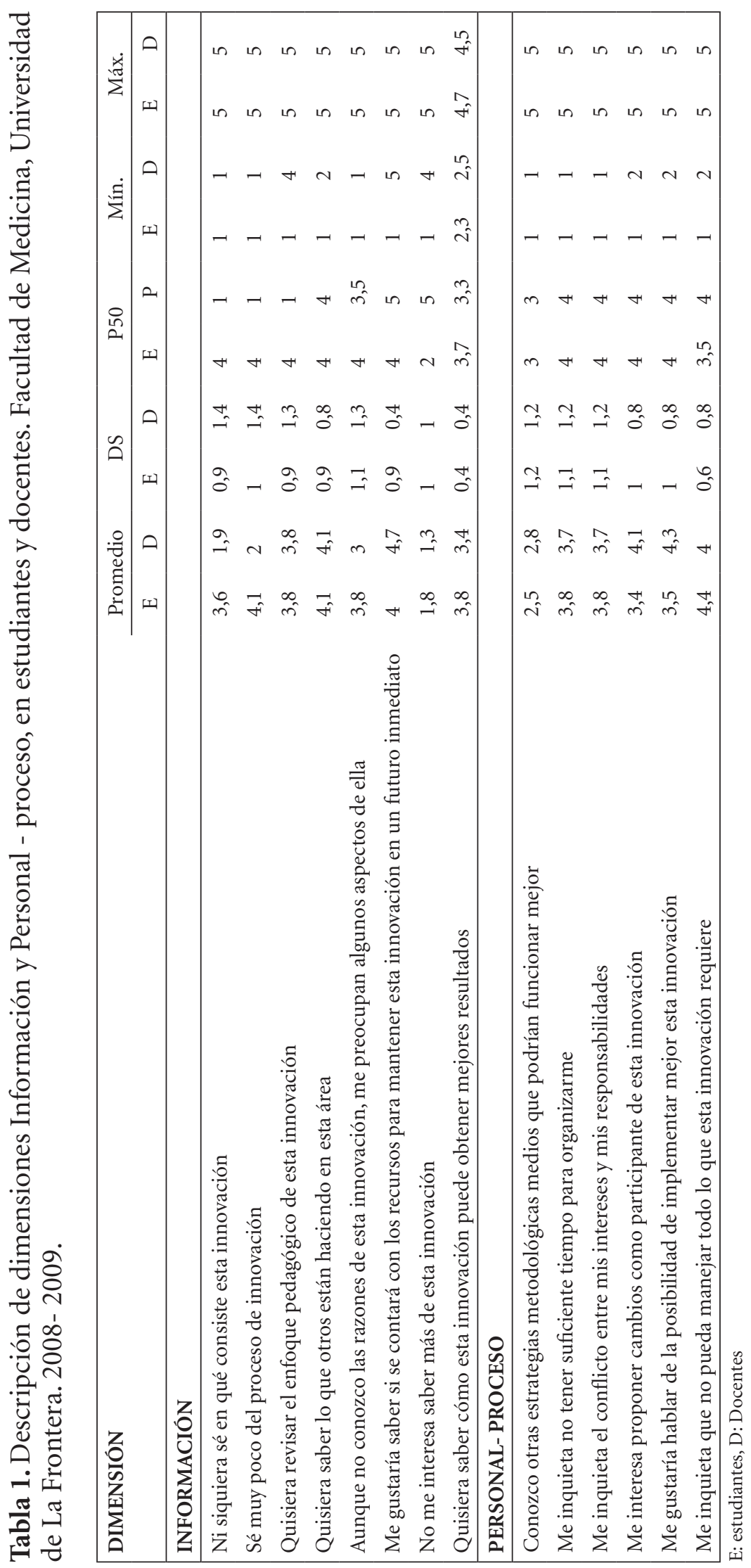




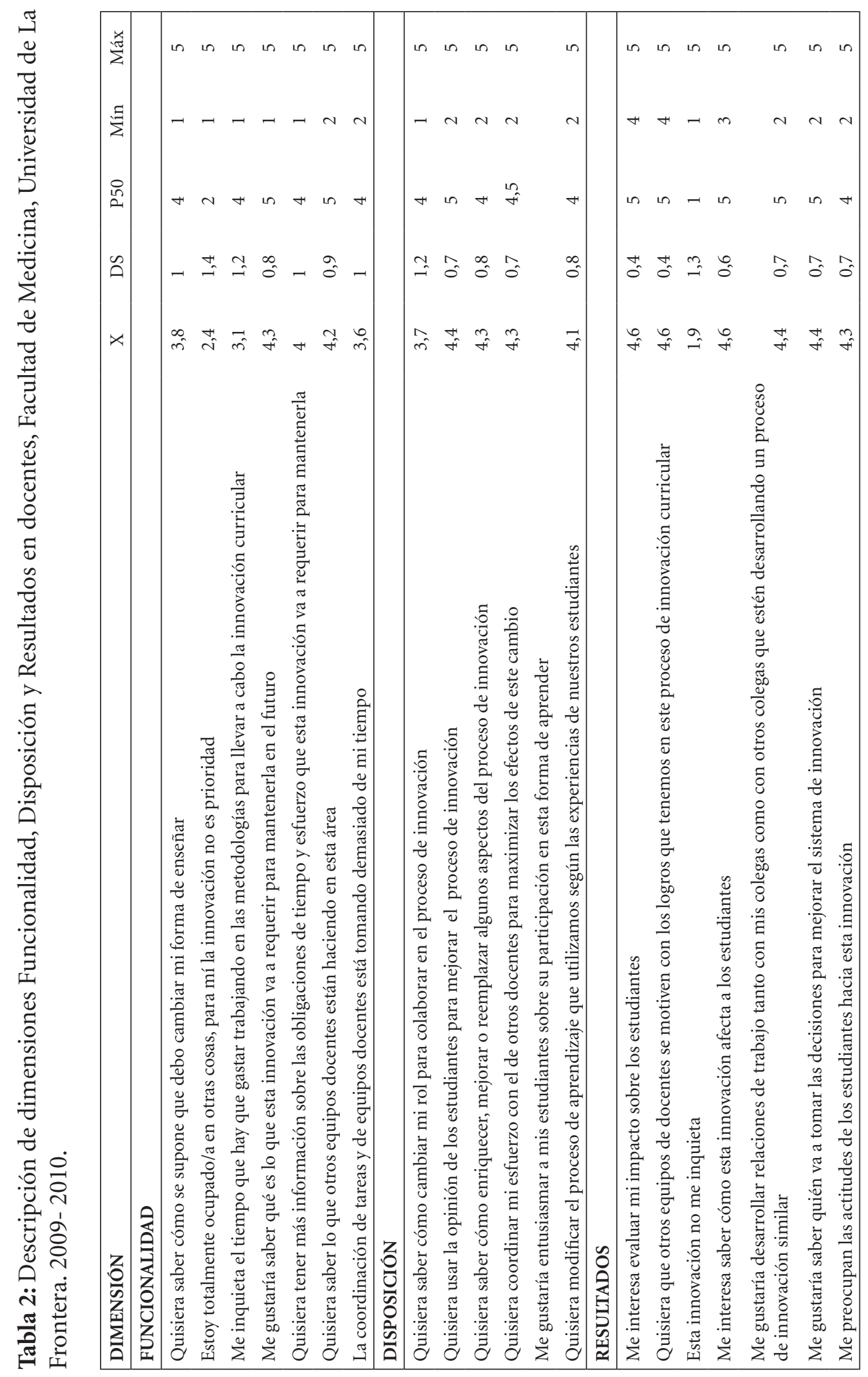


Por otra parte, se puede bifurcar en dos grandes ámbitos: rendimiento de los estudiantes y proceso educativo (15). En esta línea, las opiniones de los estudiantes se relacionan directamente con las competencias profesionales del saber (aprender a aprender), del hacer (aprender haciendo, trabajo en equipo), saber estar (compromiso social) y del aprender a aprender (creatividad) (18). De esta forma, los resultados de esta línea de investigación generan controversias entre docentes y alumnos, por atribuirle al alumnado un componente significativo de carga emocional, que las opiniones no dejan de ser fruto de sus percepciones de la realidad, y que éstas, a su vez, se ven influenciadas por sus expectativas, necesidades y factores externos (18).

Sin embargo, en la actualidad las distintas carreras del ámbito de la salud sienten que tienen la capacidad y obligación de modificar las competencias exigidas, los objetivos profesionales e, incluso, las conductas de sus egresados (19-25), avalando sus cambios en el ejercicio profesional, entorno sociocultural, acceso a la información y recursos formativos.

Así, se deben sumar los medios para que los docentes adquieran competencias en los contenidos de sus disciplinas, metodologías de enseñanza y evaluación, utilización de recursos avanzados para la búsqueda y transmisión efectiva del conocimiento, aprendizaje de destrezas y en el manejo de la administración académica (26-27).

Estudiantes y docentes están satisfechos con la experiencia del cambio curricular. El $50 \%$ de los estudiantes y la mayoría de los docentes son conocedores del cambio curricular, teniendo incorporados los contenidos abordados en el proceso enseñanza-aprendizaje.

La carrera de Tecnología Médica presentó la puntuación más baja en relación al ítem información; la carrera de Enfermería y Nutrición puntuaron favorablemente en relación a este ítem. Este resultado puede estar influenciado por el campo de acción que dichas carreras realizan.

Finalmente, aún existen dudas en el alumnado y docentes, respecto de cómo el cambio curricular afectará las competencias del estudiantado, la mejora en la innovación a través de los recursos existentes y la sustentabilidad en el tiempo. Es necesario continuar esta línea investigativa durante los años de ejercicio profesional de los egresados para evaluar objetivamente las competencias del nuevo currículo centrado en el estudiante.

\section{REFERENCIAS}

1. Millán T, Ercolano M, Pérez M, Fuentes C. Autoevaluación de habilidades clínicas básicas en médicos recién egresados de la Facultad de Medicina, Universidad de Chile. Rev Med Chil. 2007; 135(11): 1479-1486.

2. Lucarelli, E. Prácticas Innovadoras en la Formación del Docente Universitario. Educação [Internet]. 2004 [citado 4 octubre 2011]; 3(54): 503-24. Disponible en: http://redalyc.uaemex.mx/ pdf/848/84805410.pdf

3. Medina Moya JL. Práctica educativa y práctica de cuidados enfermeros desde una perspectiva reflexiva. Revista de Enfermería [Internet]. 2002 [citado 4 octubre 2011]; 15. Disponible en: http://www. uclm.es

4. Bendersky S. Educación Superior, la Reforma que viene [Internet]. 2008 [citado 4 octubre 2011]. Disponible en: www. dialoga.cl

5. Facultad de Medicina, Universidad de la Frontera. Proyecto MECESUP FRO0003. Innovación curricular en la Facultad de Medicina. Herramienta clave para responder a las demandas emergentes de la sociedad. Temuco. Chile: Universidad de La Frontera; 2003.

6. Facultad de Medicina. Planificación Es- 
tratégica de la Facultad de Medicina. Temuco. Chile. Universidad de La Frontera; 1995.

7. Bandaranayake R. How to Plan a Medical Curriculum. Med Teach. 1985; 7(1): 7-13.

8. Bandaranayake R. The Concept and Practicability of a Core Curriculum in Basic Medical Education. Med Teach. 2000; 22, (6). 560-3.

9. Herrera R. Bases para el desarrollo cualitativo de la docencia universitaria. En: Centro Interuniversitario de Desarrollo (CINDA), ed. Gestión de la docencia e internacionalización en universidades chilenas. Santiago, Chile: CINDA; 1998. p. 79-92.

10. Universidad de La Frontera. Informe de autoevaluación institucional [Internet]. Dirección de Análisis y Desarrollo Institucional. 2004 [citado 20 octubre 2011]. http://www.ufro.cl

11. Werner D. Educación superior. Evaluación y cambio curricular en Estados Unidos [Internet]. Seminario Internacional Educación Superior: nuevos currículos para los nuevos tiempos; 2006; Santiago, Chile: CNED [citado 20 octubre 2011]. Disponble en: http://cned.cl

12. Donoso S. Nuevo rol del docente: Nuevos desafíos en la docencia. Revista Calidad en la Educación. 2001; 15.

13. O’Brien K. Evaluación y cambio curricular. Revista Calidad de la Educación. 2006; 10.

14. Emmanuel E. ¿Qué hace que la investigación clínica sea ética? Siete requisitos éticos. En Lolas F, Quezada A, ed. Pautas éticas de investigación en sujetos humanos: nuevas perspectivas. Santiago, Chile: Programa regional de bioética OPS/ OMS; 2003.

15. González E. Renovación curricular en carreras de la salud. Seminario Internacional Educación Superior: nuevos currículos para los nuevos tiempos; 2006; Santiago, Chile: CNED [citado 20 octubre 2011]. Disponble en: http://cned.cl
16. Troncoso K, Hawes G. Organización y estructuración del currículum de formación profesional, la necesidad de articulación entre la formación básica y especializada [Internet]. 2011 [citado 20 octubre 2011]. Disponible en: http://www. cesuchile.cl

17. Rial A. La planificación y el diseño curricular por competencias: un reto para la educación del futuro. REDEC, Revista Electrónica de Desarrollo de Competencias [Internet] 2010 [citado 25 octubre 2011]; 10 (5). Disponible en: http:// redec.utalca.cl/index.php/redec/article/ view/38/42

18. Illesca M, Cabezas M. Satisfacción de los estudiantes en relación con la docencia y administración carrera de Enfermería. Universidad de La Frontera. Rev Educ Cienc Salud. 2006; 3(2): 82-83.

19. Anderson M. A guide to the 130 reports in this snapshot supplement to Academic Medicine. Acad Med. 2000; 75 (9 Suppl): SX-SXIV.

20. Callahan D. The goals of medicine: setting new priorities. Hastings Cent Rep. 1996; 26(6): S1-S27.

21. Maxwell S, Walley T; BPS Clinical Section Committee. Teaching safe and effective prescribing in UK General Medical School: a core curriculum for tomorrow's doctors. Br J Clin Pharmacol. 2003; 55(6): 496-503.

22. Whitcomb M. CME Reform: An imperative for improving the quality of medical care. Acad Med 2002; 77(10): 943-4.

23. Harden RM, Grant J, Buckley G, Hart IR. BEME Guide $\mathrm{N}^{\circ} 1$ : Best evidence medical education. Med Teach. 1999; 21(6): 55362.

24. Edwards M. Modern medicine and the pursuit of cure. Med Educ. 1999; 33(9): 704-6.

25. Kassebaum D, Eaglen R, Cutler E. The meaning and application of medical accreditation standards. Acad Med. 1997; 72: 807-18. 
26. Escuela de Postgrado, Facultad de Medicina, Universidad de Chile y Departamento de Educación en Ciencias de la Salud. Diploma en Docencia en Ciencias de la Salud. 2002-3 en Rosselot J. Hacia el médico que nuestros países necesitan: Énfasis en la comunicación y en la for- mación de los docentes. Rev Med Chil. 2003; 131(3): 331-337.

27. Benor D. Faculty development, teacher training and teacher accreditation in medical education: twenty years from now. Med Teach. 2000; 22: 503-12. 